\title{
SDQO and SfO, Ontologies for Spatial Data Quality Assessment
}

\author{
C. Yilmaz ${ }^{1}$, C. Comert ${ }^{1}$, D. Yildirim ${ }^{1}$ \\ ${ }^{1}$ KTU, Engineering Faculty, Dept. of Geomatics Engineering, 61080 Ortahisar, Trabzon Turkey - (cemre.yilmaz, ccomert, \\ dyildirim)@ktu.edu.tr
}

KEY WORDS: Geo-ontology, specification ontology, spatial data quality ontology, spatial data quality management.

\begin{abstract}
:
Spatial quality assessment is based on the conformance of data to its specifications or fitness for users' purpose. These specifications and the users' purposes include the rules and constraints that a dataset should comply with. Assessing the compliance of data to the rules is still an active research subject and rule-based approach is the common method. For the efficient rule-based system implementation, it is desired to automate assessment process with a domain-independent and web-based approach. Reasoning capability and re-usability of semantic web components are expected to promote efficient implementation. In literature, many domains such as agriculture, music, Linked Data and geospatial domain etc. apply ontology-based methods for quality management. There is a need to model geospatial quality concepts and rules in a domain-independent way to automate the quality management process. In our model of rule formalism, we use Web Ontology Language (OWL) and Semantic Web Rule Language (SWRL). We devise two types of ontologies. These are; the specification ontologies (SfO) and the Spatial Data Quality Ontology (SDQO). SfO is to be created by domain experts/users to define rules according to specifications. SDQO is responsible with quality assessment; it is domain independent and makes assessment based on the rules defined by any SfO for the related domain. The quality elements are domain and toposemantic consistency that assessed by SWRL. In this paper, the design considerations of the ontologies for quality assessment are explained with an example.
\end{abstract}

\section{INTRODUCTION}

High quality spatial data is essential in providing better analyses and making better decisions involving such data. There have been studies such as ISO (2013), Zaveri et al. (2015), Fonte et al. (2017) to categorize and define the quality concepts, quality evaluation methods and solutions for producing better data.

In the modern age of web, Semantic Web ("meaningful web") components such as ontologies are used for Rule-based system implementation because of expressivity, reasoning capability and re-usability. This is still an active research theme with recommended, accepted and upcoming standards; SWRL (W3C, 2004), SPARQL Inferencing Notation (SPIN) (W3C, 2011), Rule Interchange Format (RIF) (W3C, 2013) and a newer standard for schema validation Shapes Constraint Language (SHACL) (W3C, 2017).

Mostafavi et al. (2004), Fürber and Hepp (2011), Debattista et al. (2016), Degbelo (2012), Geisler et al. (2016), Zhu (2014) and Nash et al. (2011) are the examples to the studies that use ontologies for data quality management purposes.

Mostafavi et al. (2004) propose ontology based method to detect inconsistent spatial data for Canada topographic data specifications with the help of Prolog rules. Fürber and Hepp (2011) propose data quality management (dqm) ontology for assessment of web of data. Debattista et al. (2016) developed daQ (Dataset Quality Ontology) for data quality standardization purpose especially for linked data. They also create a linked data quality framework called Luzzu. Degbelo (2012) creates an ontology design pattern (ODP) to model a specific quality problem of Semantic Sensor Network. This ontology has a specific model to solve a recurrent problem in the Semantic Sensor area. Geisler et al. (2016) proposes ontology-based approach to test quality metrics of data streams. Zhu (2014) propose ontology-based quality assessment framework for a specific clinical domain (organ transplant).

In addition to quality management with OWL, SWRL rules are used in several studies for quality assessment process. (Wang et al., 2005); (Cheng et al., 2008); (Keßler et al., 2009); (Zhu, 2013); (Cherfi et al., 2017); (Varadharajulu et al., 2017); (Mobasheri, 2017); (Homburg and Boochs, 2019) can be given as example.

Wang et al. (2005) developed a system to detect inconsistent spatial data with the help of SWRL rules in a specific domain. Homburg and Boochs (2019), Varadharajulu et al. (2017), and Mobasheri (2017) propose rule-based approaches including SWRL rules for domain dependent solutions. Varadharajulu et al. (2017) design a framework to check the consistency of the transportation data against the rules that are created with SWRL.

In these studies, SWRL rules are used with a domain dependent quality management framework. Mobasheri (2017), proposes a rule -based system to increase the Quality of the OSM data with rules created by SQWRL.

Besides the previously explained studies, Nash et al. (2011) design a framework for the automatization of specification rules in agriculture domain with implementation of geospatial rules as Interchangeable Rule Format (RIF) and proposes, GeoRIF. The ontology they devised is specific to the agriculture regulations.

It is desired to make a robust framework that can be applicable to spatial data, independent from the domain. This will reduce redundancy and increase interoperability. Hence, there is a need to create ontology for the designed framework. The components and details of the framework is out of the scope of this paper. The main focus is the explanation of devised ontologies. These are; Specification Ontology (SfO) and Spatial Data Quality Ontology (SDQO). Following sections describe SDQO and SfO and briefly explain how to use them for quality assessment with an example rule.

2.

\section{ONTOLOGIES FOR SPATIAL DATA QUALITY} MANAGEMENT

\subsection{Ontology Design}

Initial step to create ontology for quality management is to define the concepts. For this purpose, motivating scenario and 
competency questions are defined. Followingly, existing ontologies are researched for both geospatial and data quality management concepts. The following sections explain the motivating scenario and questions, ontologies for quality management and geo-ontologies respectively.

\subsubsection{Motivating Scenarios and Questions}

Spatial data producers want to produce data compliant to the rules in the regulations/specifications. Producers should formalize the rules, assess the data and have the quality result as a report.

Data producers can select the rules that should be applied to a specific spatial task in a domain. Formalized rules for a domain can be stored and reusable for further assessments.

- Which instances of tested class have data quality problems according to predefined data quality rules?

- Which instances of tested class have spatial relation problems with the specified ones? e.g. mustBeWithin, mustNotCross.

- Which instances of tested class have cross relation with the instances of another class when it is forbidden? (must not cross)

- Which instances of tested class overlap with the instances of the same class when it is forbidden? (must not overlap)

- Which instances of tested class with the type of Polygons/Lines overlaps any other feature in the same class when it is forbidden? (must not overlap with)

- Which instances of tested class are spatially within any instance from the second feature class when it is forbidden? (must not be within)

- Which instances of the tested class is not within any instance of the second class when it is forbidden? (must be within)

- Which instances of tested class have domain consistency problems?

$\circ$ Which instances of tested class have attributes different from of a constant value while it is forbidden?

- Which instances of tested class have "null" data value for its attributes while it is forbidden?

- Which instances of tested class have an attribute greater than a specified one while it is not allowed?

- What is the percentage of the erroneous instances in the tested data?

\subsubsection{Geospatial Ontologies}

Mostly used ontologies for geospatial domain are; GeoNames (GeoNames Team, 2006), W3C Geo (W3C Semantic Web Interest Group, 2004), GeoOWL (W3C Geospatial Incubator Group, 2007) and OGC GeoSPARQL (OGC, 2012). The GeoNames Ontology and W3C Geo support only point type geometries. GeoOWL, the updated model of W3C Geo, is created compatible with GeoRSS Feature Model. Only GeoSPARQL supports other geometry types such as polygon and lines and basic spatial relations. The GeoSPARQL ontology has been selected for defining geospatial concepts and relations shown in Figure 1. Its classes are used as superclasses to the classes in the designed ontologies.

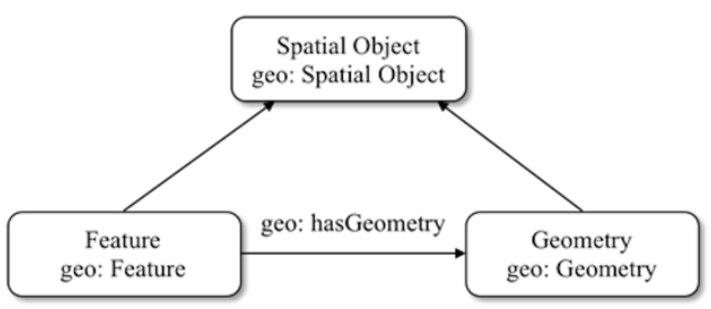

Figure 1. GeoSPARQL classes.

\subsection{Devised Ontologies}

In this study two types of ontologies are introduced. The SDQO ontology, contains the necessary rules and the concepts related with the quality assessment. The SfOs are simple ontologies, developed keeping in mind the reusability of rules with different kinds of datasets. There can be one or more SfOs (eg. for different scales) for each institution. Devised ontologies and their relations are shown on Figure 2. This section continues with the subsections to explain SDQO and SfO.

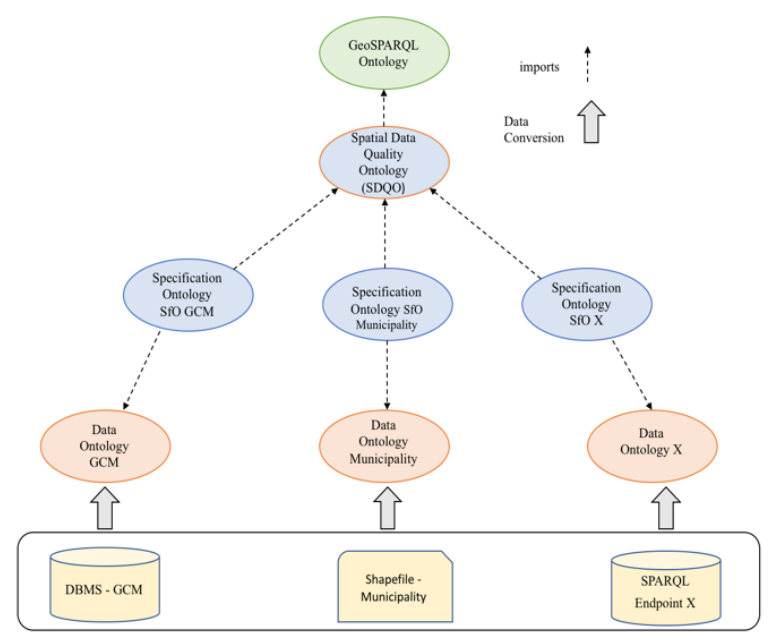

Figure 2. Ontologies and their relations.

\subsubsection{SDQO}

According to the set of rules depending on appropriate and chosen elements for data quality, SDQO is also responsible of processing and integrating data quality elements with associated procedures and implementing the procedures in accordance with the geospatial data and the quality elements. SDQO prepares the resulting spatial data quality ontology which relates data quality results with tested data and prepares it for queries or publishing.

While creating the SDQO, terminologies which are used in other studies are considered. Although, all ontologies are created with the same intention, quality management, they are specific to different application domains. They have some common concepts such as "Quality Dimension", "Quality Metric" or "Quality Result".

In SDQO, there are three top classes directly below owl:Thing, one for data, one for data quality elements, and one for data quality results and processes. These are ogc:SpatialObject, sdqo:DataQualityElement, sdqo:DataQualityResult, respectively as shown on Figure 3. 


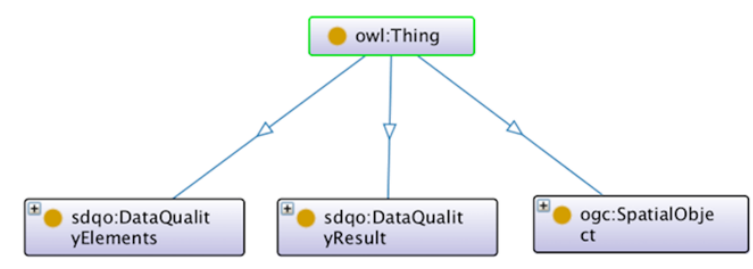

Figure 3. SDQO ontology, top classes

In SDQO, ogc:Feature has three direct subclasses, other than the ones from imported ontologies. These are sdqo:GeomClassifiedFeature, sdqo:FixedRefFeature and sdqo:RestrictedFeature. These classes are represented in Figure 4.

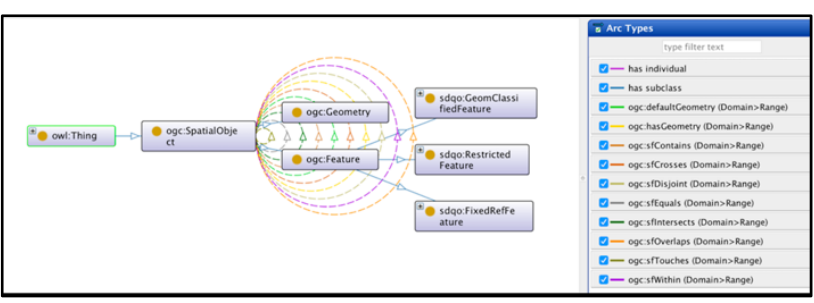

Figure 4. ogc-Feature Class with subclasses.

sdqo:RestrictedFeature has four direct subclasses, as shown in Figure 5. These are sdqo:InterObjectPrRF, sdqo:IntraDataPrRF, sdqo:IntraObjectPrRF and sdqo:InterDataPrRF. Rules and given subclass relations determine which features these classes have. The labels and the descriptions are listed in Table. "Intra" classes are for restrictions within a single class and "Inter" classes are for restrictions in class pairs. sdqo:RestrictedFeature has the label "Features according to the property restrictions".

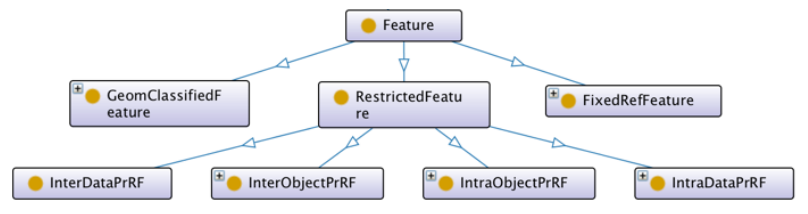

Figure 5. RestrictedFeature class and its subclasses.

sdqo:GeomClassifiedFeature is defined to classify features classified according to their geometries.

It has three subclasses, sdqo:CalcPoly, sdqo:CalcLine and sdqo:CalcPoint, declared to be owl:disjoint. Any feature that is associated with a geometry that has a valid ogc:asWKT value, is automatically put in the correct one according to the ogc:asWKT value, using a SWRL rule. This information is to be used in other rules.

sdqo:FixedRefFeature has OWL named individuals to be used in rules as reference markings. For instance, attribute tests can make use of reference individuals.

sdqo:RestrictedFeature: "Features according to the property restrictions".

sdqo:RestrictedFeature has four direct subclasses, sdqo:InterObjectPrRF, sdqo:IntraDataPrRF, sdqo:IntraObjectPrRF and sdqo:InterDataPrRF. Rules and given subclass relations determine which features these classes have. The labels and the descriptions are listed in Table 1. "Intra" classes are for restrictions within a single class and "Inter" classes are for restrictions in class pairs.
Table 1. SDQO superclasses

\begin{tabular}{|l|l|}
\hline Class & rdfs:label \\
\hline sdqo:InterObjectPrRF & $\begin{array}{l}\text { Feature restricted wrt } \\
\text { relations between classes }\end{array}$ \\
\hline sdqo:InterDataPrRF & $\begin{array}{l}\text { Feature restricted by } \\
\text { attributes between classes }\end{array}$ \\
\hline sdqo:IntraDataPrRF & $\begin{array}{l}\text { Feature restricted by } \\
\text { attributes within class }\end{array}$ \\
\hline sdqo:IntraObjectPrRF & $\begin{array}{l}\text { Feature restricted wrt } \\
\text { relations within class }\end{array}$ \\
\hline
\end{tabular}

sdqo:DataQualityElement is defined as abstract class to classify the elements of data quality. It has subclasses as follows,

sdqo:GeometricAccuracy has sdqo:GeometryValidity as a subclass.

sdqo:TopoSemanticConsistency is a subclass to sdqo:SemanticAccuracy and sdqo:LogicalConsistency. sdqo:LogicalConsistency also has sdqo:DomainConsistency.

sdqo:DataQualityResult is defined as abstract class to classify the results of data quality assessment.

SDQO has the following own datatype properties. Several of them are to be used as a super property to data properties in SfOs.

Other datatype properties are schema:startTime and OGC datatype properties such as ogc:asWKT.

sdqo:errorCode datatype property is defined to give a code to results to identify the problem of data.

sdqo:hasErrorCode datatype property is defined to relate data with the resultant sdqo:errorCode.

sdqo:dataHasErrorWithCode datatype property connects erroneous data to error codes.

Every feature must have sdqo:featureID datatype property which is to be unique to every feature.

sdqo:hasMessage datatype property connects results and processes to error messages.

sdqo:elementHasMessage datatype property is a shortcut from elements to error messages (sdqo:hasResult and sdqo:hasMessage).

There are SDQO datatype properties for attribute tests such as sdqo:dataProp01.

sdqo:subnr property is the main data property for assigning the classes to be tested with the subclass number. It has subproperties such as sdqo:subnrOverlap and sdqo:subnrMustWithin. These subproperties are used in defining top classes in SfO.

sdqo:hasQueryString datatype property is for SPARQL query strings.

While sdqo:resultForData object property create a relationship between quality results and data, sdqo:hasResult relates data quality elements to results. sdqo:dataHasResult is inverse property to sdqo:resultForData.

Furthermore, GeoSPARQL object properties such as ogc:sfOverlaps are used for relations between spatial classes. ogc:sfIntersects is declared to be a super property to intersection type properties such as ogc:sfOverlaps. SDQO as in Protégé ontology editor is shown in Figure 6. 


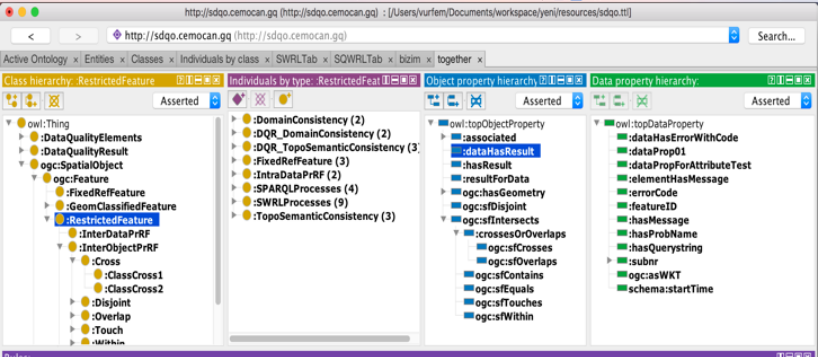

Figure 6. SDQO in Protégé ontology editor.

\subsection{SfO, Specification Ontology}

$\mathrm{SfO}$ is designed to be manageable by domain experts to be even without Semantic Web expertise. A GUI is created for users to input rules according to the specifications. Once rules are defined by the help of GUI, an OWL file (SfO) is created as a result of translations from GUI to CSV and OWL files respectively. The translations and the assessment framework are out of the scope of this study. The SfO and its components will be explained in detail.

A SfO has the class hierarchy reflecting the associated specifications. Furthermore, these specification hierarchy classes have superclasses, still in SfO. These classes are created to deanonymize the restrictions. They are the top classes with SfO's IRI. They are subclasses to SDQO classes and establish the connection to SDQO. Furthermore, these top classes should have associated geometries under the appropriate simple features class, such as sf:Polygon or sf:Linestring.

SfOs directly import SDQO and the directly or indirectly import data ontologies associated with that $\mathrm{SfO}$ or other SfOs.

With $\mathrm{SfO}$, more general relations are translated into is-a relations in the scope of SfO, if possible. The feature pairs causing the errors are identified. Below, a sample translation is given.

\begin{tabular}{l|l} 
Specification rule & Implementation \\
\hline Features in class A & A is a subclass of C, an SfO class; B is \\
must not overlap & $\begin{array}{l}\text { a subclass D, an SfO class; these are } \\
\text { with features in } \\
\text { class B }\end{array}$ \\
$\begin{array}{l}\text { take part in SWRL rules establishing the } \\
\text { relevant error properties involving } \\
\text { faulty features in A, B and forbidden } \\
\text { overlaps relation. }\end{array}$ \\
\hline
\end{tabular}

SfOs define specification classes and generic classes for data to be tested. SfO generic classes are defined according to the spatial relations related to data. Spatial relations should be defined between feature classes. In specifications, a feature class might have topological relations with; itself, a feature class, more than one feature class.

Specification classes are SfO classes such as "sfo:Road" and "sfo:Building", that exist in the specifications. They can have subclasses; sfo:PermanentLake can be a subclass to sfo:Lake.

Examples include sfo:ClassOvf01, sfo:ClassOvf02, etc. and sfo:ClassOvs01, sdqo:ClassOvs02, etc. . Here Ov is for Overlaps, following $f$ is for 'first', 's' is for 'second'. The numbers represent the subclass numbers, the order in pairs of specification classes. A path of subclass relations in a SfO is shown in Figure. 7.

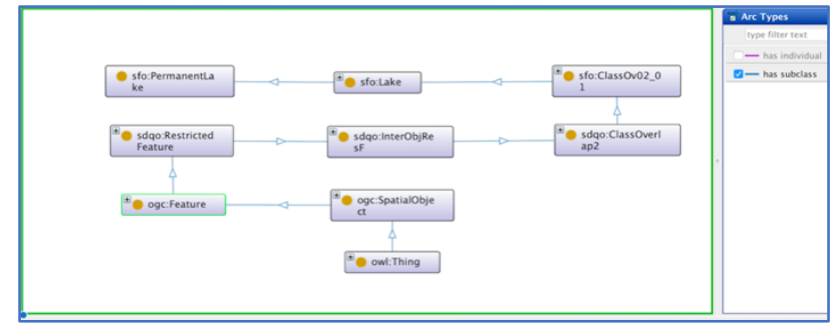

Figure 7. A path of subclass relations in a $\mathrm{SfO}$

\subsection{SDQO and SfO relation}

The data quality elements are linked to individuals for processes and results. These individuals are used in SWRL rules and SPARQL queries. Classes for features with constraints are defined. SfOs are devised to define which restrictions will be applied to dataset according to specifications by a domain expert. They are mostly hierarchical. SfOs import SDQO and data ontologies. The data ontologies are likely to have some faults. The system should be robust and stay consistent. The system is designed to be robust and user-friendly, therefore usable. The common parts of the SfOs are moved to SDQO. Most of the rules are in the SDQO. The use of SDQO enables domain-independent, easy-to-update quality assessment with the SfOs. Especially with an ontology editor, it is expected that domain experts can quickly understand how to manipulate and update their SfOs, when necessary. SfOs typically do not use the whole capability of the SDQO. When the rulesets change, even without needing to change the SDQO significantly, it is expected that the new SfO can be implemented. Thanks to the prevailing OWA, SDQO is easy-to-update, when necessary. With OWA, truth of statements does not change.

Case 1: Geometric classes with Forbidden relation (Inter-object Must Not).

Example: "c1, c2, Forbidden, Crosses, class1, class2|class3, $6210.12 "$

Meaning: "class1 is of line-type, class 2 and class 3 are of polygon-type, features in class 1 cannot have the Crosses relation with the features in the classes class 2 and class 3 " 6210.12 is the timestamp, which is the same throughout the process (the minimal one).

Two generic classes are created in the SfO; they are set as subclasses of the relevant classes in SDQO. If there are already six "c1,c2, Forbidden,Crosses" pairs, the created classes are sfo:ClassCrf07 and sfo:ClassCrs07. Here "Cr" stands for "Crosses", "f" stands for "first" and "s" stands for "second". Restrictions are among anonymous classes in OWL. sfo:ClassCrf07 is set as a subclass of SDQO class sdqo:ClassCross1 and sfo:ClassCrs07 is set as a subclass of sdqo:ClassCross2.

The subclass relations are established. sfo:class1 is created as a subclass of sfo:ClassCrf07. sfo:class 2 and sfo:class 3 are created as subclasses of sfo:ClassCrs07. A sample for relevant class hierarchies are given below with the associated SWRL rule. It is also shown in Figure 8. " $<$ " denotes the subclass relation. Case 1 type cases ("Inter-object Must Not") are easily updated by updating the subclass relations of the specification classes or creating new classes with the appropriate subclass relations. 
SWRL rules for this kind of restriction is as follows, crossesOrOverlaps(?x, ?y)^ CalcLine(?x) $)^{\wedge}$ CalcPoly(?y) $\quad$-> sfCrosses(?x, ?y)

RestrictedFeature(?x) $\wedge$ ogc:hasGeometry(?x, ?g) $\wedge$ ogc:asWKT(?g, ?w) ^ swrlb:contains(?w, "LINESTRING") -> CalcLine(?x)

RestrictedFeature(?x) $\wedge$ ogc:hasGeometry(?x, ?g) ogc:asWKT(?g, ?w) ^ swrlb:contains(?w, "POLYGON") -> CalcPoly(?x)

Sample class hierarchy paths:

sfo:Road < sfo:ClassCrf07 < sdqo:ClassCross $1<$ sdqo:Cross $<$ sdqo:InterObjectPrRF $<$ sdqo:RestrictedFeature $<$ ogc:Feature $<$ ogc:SpatialObject $<$ owl:Thing

sfo:Road $<$ sfo:ClassCrf07 $<$ [ sdqo:subnrCross $=7$ ]

sfo:Building $<$ sfo:ClassCrs $07<$ sdqo:ClassCross $2<$ sdqo:Cross $<$ sdqo:InterObjectPrRF $<$ sdqo:RestrictedFeature $<$ ogc:Feature $<$ ogc:SpatialObject $<$ owl:Thing

sfo:Building $<$ sfo:ClassCrs07 $<[$ sdqo:subnrCross $=7]$

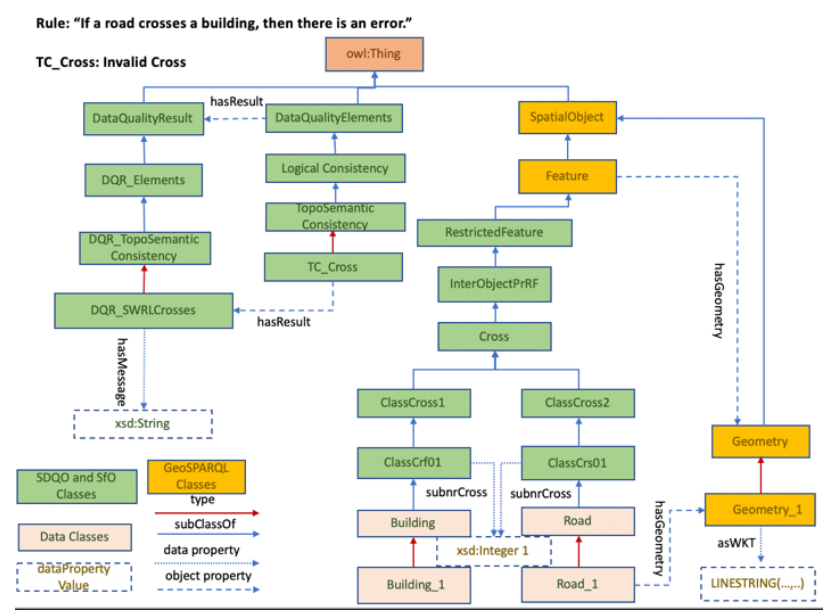

Figure 8. Rule: "If a road crosses a building, then there is an error." SfO and SDQO class relations.

\section{CONCLUSION}

The aim of this study was to create ontologies that can be used for spatial data quality assessment process. To support reusability, domain independence, extensibility and spatial quality rules two types of ontologies are devised. SfO is created by the domain experts to represent rules. Once a company/institution or a user create the SfO for its domain (agriculture, transportation etc.) then this ontology can be saved to assess data against those rules in SfO. SDQO ontology is responsible for the assessment part. It has mainly three classes; ogc:Feature class from GeoSPARQL ontology, sdqo:QualityElement and sdqo:QualityResult. SfO separate the rule creation part from the assessment part. SDQO has SWRL rules for finding inconsistent instances. As a result, the devised ontologies can be used for data quality management independent from domain and the concepts for spatial rules make it possible to represent spatial problems.

\section{REFERENCES}

Cheng, G., Du, Q., Ma, H., 2008. The Design and Implementation of Ontology and Rules Based Knowledge Base for Transportation, in: 2008 International Conference on Computer Science and Software Engineering. Presented at the 2008 International Conference on Computer Science and Software Engineering, IEEE, Wuhan, China, pp. 1035-1038. https://doi.org/10.1109/CSSE.2008.1405

Cidália Costa Fonte, Vyron Antoniou, Lucy Bastin, Jacinto Estima, Jamal Jokar Arsanjani, Juan-Carlos Laso Bayas, Linda See, Rumiana Vatseva, 2017. Assessing VGI Data Quality, in: Mapping and the Citizen Sensor.

Debattista, J., Auer, S., Lange, C., 2016. Luzzu -- A Framework for Linked Data Quality Assessment, in: 2016 IEEE Tenth International Conference on Semantic Computing (ICSC). pp. 124-131. https://doi.org/10.1109/ICSC.2016.48

Degbelo, A., 2012. Short Paper: An Ontology Design Pattern for Spatial Data Quality Characterization in the Semantic Sensor Web. Proc 5th Workshop Semantic Sens. Netw. 6-11.

Fei Wang, Stephan Mäs, Wolfgang Reinhardt, Admire Kandawasvika, 2005. Ontology Based Quality Assurance for Mobile Data Acquisition. Presented at the Informatics for Environmental Protection - Networking Environmental Information.

Fürber, C., Hepp, M., 2011a. Towards a vocabulary for data quality management in semantic web architectures, in: Proceedings of the 1st International Workshop on Linked Web Data Management - LWDM '11. p. 1. https://doi.org/10.1145/1966901.1966903

Geisler, S., Quix, C., Weber, S., Jarke, M., 2016. Ontology-Based Data Quality Management for Data Streams. J. Data Inf. Qual. 7, 1-34. https://doi.org/10.1145/2968332

GeoNames Team, 2006. GeoNames Ontology.

Homburg, T., Boochs, F., 2019. Situation-Dependent Data Quality Analysis for Geospatial Data Using Semantic Technologies, in: Abramowicz, W., Paschke, A. (Eds.), Business Information Systems Workshops. Springer International Publishing, Cham, pp. 566-578. https://doi.org/10.1007/978-3030-04849-5_49

ISO, 2013. 19157, Geographic Information-Data Quality.

Keßler, C., Raubal, M., Wosniok, C., 2009. Semantic Rules for Context-Aware Geographical Information Retrieval, in: Barnaghi, P., Moessner, K., Presser, M., Meissner, S. (Eds.), Smart Sensing and Context. Springer Berlin Heidelberg, Berlin, Heidelberg, pp. 77-92. https://doi.org/10.1007/978-3-64204471-7_7

Lingkai Zhu, 2014. SemDQ: A Semantic Framework for Data Quality Assessment. University of Waterloo, Waterloo, Ontario, Canada.

Mir-Abolfazl Mostafavi, Geoffrey Edwards, Robert Jeansoulin, 2004. An ontology-based method for quality assessment of spatial data bases. Presented at the Third International Symposium on Spatial Data Quality, Bruck an der Leitha.

Mobasheri, A., 2017. A Rule-Based Spatial Reasoning Approach for OpenStreetMap Data Quality Enrichment; Case Study of Routing and Navigation. Sensors 17, 2498. https://doi.org/10.3390/s17112498 
Nash, E., Wiebensohn, J., Nikkilä, R., Vatsanidou, A., Fountas, S., Bill, R., 2011. Towards automated compliance checking based on a formal representation of agricultural production standards. Comput. Electron. Agric. 78, 28-37. https://doi.org/10.1016/j.compag.2011.05.009

OGC, 2012. Geosparql-a Geographic Query Language for RDF Data.

Samira Cherfi, Fayçal Hamdi, Philippe Rigaux, Virginie Thion, Nicolas Travers, 2017. Formalizing Quality Rules on Music Notation-an Ontology-based Approach. Presented at the International Conference on Technologies for Music Notation and Representation (TENOR'17), A Coruña, Spain.

Varadharajulu, P., Arnold, L., McMeekin, D.A., West, G., Moncrieff, S., 2017. SWRL Rule Development to Automate Spatial Transactions in Government, in: Grueau, C., Laurini, R., Rocha, J.G. (Eds.), Geographical Information Systems Theory, Applications and Management. Springer International Publishing, Cham, pp. 122-142. https://doi.org/10.1007/978-3319-62618-5_8

W3C, 2017. Shapes Constraint Language (Shacl).

W3C, 2013. RIF- Basic Logic Dialect.

W3C, 2011. SPIN - Overview and Motivation.

W3C, n.d. SWRL: A Semantic Web Rule Language Combining OWL and RuleML.

W3C Geospatial Incubator Group, 2007. GeoOWL.

W3C Semantic Web Interest Group, 2004. Basic Geo (WGS84 lat/long) Vocabulary.

Zaveri, A., Rula, A., Maurino, A., Pietrobon, R., Lehmann, J., Auer, S., 2015. Quality assessment for Linked Data: A Survey: A systematic literature review and conceptual framework. Semantic Web 7, 63-93. https://doi.org/10.3233/SW-150175

Zhong Hu, Y., Zhong, B., Luo, H., Meng Hu, H., 2013. The regulation constraint modeling and semantic inferring in construction quality checking. Struct. Surv. 31, 387-400. https://doi.org/10.1108/SS-10-2012-0033 